\title{
Special Breathing Exercises in Persons with SCI and Evaluate their Effectiveness by Using X-ray of Lungs and Other Tests
}

\author{
By Artem Vetkasov* \\ Blanka Hoskova ${ }^{\dagger}$
}

From clinical practice and research it is known that breathing exercises have a positive effect in people with spinal cord injury (SCI). The purpose of this study was to perform special breathing exercises in persons with PM and evaluate their effectiveness by using X-ray of lungs and other tests. Sample consisted of 15 respondents with SCI, 8 men and 7 women, mostly middle-aged and elderly. Intervention program has lasted six months, with the frequency of exercise five times a week, in the range of 20-30 minutes. The study has included:: 1 ) Chest X-Ray in a sitting position during inhalation and exhalation; 2) chest excursion; 3) determination of forced vital capacity $(F V C)$ and forced expiratory volume during first second (FEVI ); 4) measurement of respiratory rate. Pretest $X$-ray showed that the difference in the movement of the lower ribs during inhalation and exhalation was 2-35 $\mathrm{mm}$. Measurement of chest circumference showed a limitation of chest expansion related to normal values corrected with age and sex. Tetraplegics FVC decreased by 30-50 \% compared with the values of healthy population and paraplegics FVC reduced by about $80 \%$. Respiratory rate for all respondents with SCI was 13 to 22 breaths / min. After six months, we repeated the tests. X-ray examination showed that the difference in the movement of the lower ribs during inhalation and exhalation has increased by $49 \%$ (6 to $45 \mathrm{~mm}$ ). Circumference of chest during inspiration increased by $3.5 \%$ and chest circumference during exhalation decreased by 1.27\%. Onesecond vital capacity increased by $5.68 \%$ and forced vital lung capacity by $7.61 \%$. Respiratory rate decreased on average by $16.22 \%$. In this study, by using X-ray and other tests, we have noted the objective influence of breathing exercises on the respiratory muscles in persons with SCI.

${ }^{*}$ PhD Student, Sport Charles University, Czech Republic.

${ }^{\dagger}$ Docent, Sport Charles University, Czech Republic. 


\section{Introduction}

Spinal cord injury (SCI) often leads to impaired breathing. In most cases, such severe respiratory complications lead to morbidity and sometimes even death. However, in the last few years there has been extensive work examining ways to restore this vital function after experimental spinal cord injury. In addition to finding strategies to rescue breathing activity, many of these experiments have also yielded a great deal of information about the innate plasticity and capacity for adaptation in the respiratory system and its associated circuitry in the spinal cord.

People with SCI frequently experience a range of complications. Respiratory dysfunction (Chen, 1990), pain (Dyson-Hudson, 2004; Gironda, 2004), muscle fatigue (Rodgers, 2003), and pressure ulcers (McKinley, 1999; Thorfinn, 2002) are among the most common complaints. A major cause of morbidity and mortality in these people is long-term respiratory complication in the form of pneumonia or atelectasis, with pneumonia being the leading cause of their deaths (Jackson, 1994).

Many factors can contribute to poor lung function, including smoking habits, surgical history, hazardous occupational or environmental exposure, asthma, allergies, chronic obstructive pulmonary disease, and obesity. Additionally, the connection between posture and lung performance has been proved significant (Chen, 1990; Hobson, 1992; Baydur, 2001; Lalloo, 1991).

After an injury at a high point on the SCI, the muscles responsible for breathing are paralysed or weakened. This weakness reduces the volume of the lungs (lung capacity), the ability to take a deep breath and cough, and puts them at greater risk of lung infection. Just like other muscles of the body, it's is possible to train the breathing (respiratory) muscles to be stronger; however, it is not clear if such training is effective for people with SCI.

Study of Siska Van Hout et.al (2006) says that impaired respiratory muscle function and increased risk of respiratory complications have been frequently mentioned as an indication for respiratory muscle training (RMT) in a person with SCI. In addition, it is well established that people with quadriplegia have reduced physical capacity due to the loss of motor function in their lower and/or upper limbs and the relatively inactive lifestyle associated with the injury. In addition, respiratory muscles might partake in non-ventilatory functions during exercise. Therefore, it is assumable that exercise performance might benefit from RMT in people with SCI.

The aim of this paper is to evaluate effectiveness of breathing exercises in sitting position, in wheelchair, on postural stereotype and assessment of further objective indicators. We have decided that it would be appropriate to try to do a more objective and complex preview of this issue. Because none of these studies, which we discussed, does not offer an objective view to influencing of the breathing muscles breathing exercises, we think that investigation of this problem can bring a different quality in assessment of breathing exercisesand thus improve the prevention and treatment of respiratory system in patients with PM. 


\section{Methodology}

The study lasted for six months, from April 2013 untill September 2013. Five days a week with a frequency in the range 20-30 minutes.

Fifteen subjects with SCI participated in this study:

8 men and 7 women: high tetraplegia (C4 and C5) - 3 persons, low tetraplegia (C6 - C8) - 6 people, high paraplegia (Th1 - Th6) - 2 persons, low paraplegia (Th7 and below) - 4 people. The subjects were between 24 and 60 years of age. On average, each person was 3-15 years after injury. Four persons out of fifteen smoked, on average 23.5 years.

Within the study tests were carried out, which included:

- X-ray examination of the lungs in a sitting position - we observed differential movement of the lower ribs on the inhale and exhale (Pic. 1).

- Excursion chest - chest circumference was measured with a measuring tape - in front of the nipples (men) or of mezosternální (women) and back under the lower angles of the blades. Circumference was measured at mid breath position, then after a maximum inspiration and then after maximal expiration.

- Spirometry parametrs, forced vital lung capacity (FVC) and onesecond forced expiratory (FEV1) were determined by a spirometric system Micro Diary Card (Micro Medical Ltd., UK).

- Measurement of respiratory rate.

\section{Respiratory Muscle Training}

The Respiratory Muscle Training allows complete lung expansion, the movement of the ribs at all levels and directions. It affects the development of strong lungs and is a sufficient supply of oxygen during controlled cleansing of the body from carbon dioxide. Excessive amounts of carbon dioxide in the bloodstream are often given in connection with mental fatigue, physical fatigue and neurasthenia. It improves the work of the diaphragm, relaxes shoulders, trapezius muscles and has a positive effect on posture.

1. "Bellows": Rapid breath - rapid exhale, pushes the abdominal and diaphragm. Initially recommended slower breathing (one breath for 2 sec.) and then adding time and speed of repition. Begin with 5 rounds. Number of breaths or breathing cycle will depend on the lungs and their abilities. After you can handle basic exercises every day add two to three rounds, until finally you reach the number of 60 laps per session. Soon as you detect any sign of fatigue or hyperventilation, stop exercising, and do not access to it until the next day when you start with a lower number of rounds. 
2. Inhale, then exhale again and then without exhale one more extra breath - hold your breath for about 3 seconds - exhale, exhale and a third time to exhale completely empty the lungs - again, holding the breath for 3 seconds and pressing on the diaphragm.

3. "Strong hug": arms raised ahead, bent at the elbows, the gap between the fingers about 5 inches apart - rapid breath at that draw up arms to the body, as we would like someone to give strong hug - smooth exhale and return to the starting position.

4. "Rowing": breath in, hands stretched forward - exhale, the shoulder blades together, arms bent, elbows back, chest tighten.

5. "Butterfly": arms raised ahead, bent at the elbows, shoulder blades together. Push the arms together slowly as you squeeze your chest in the middle. Exhale during this part of the motion and hold the contraction for a second. Breath in, return back to the starting position slowly as you inhale until your chest muscles are fully stretched.

6. "Push and pull" (on each hand): inhale, hand tighten, as if to push the wall - exhale, bend the elbow, as if you wanted to draw something.

7. Arms back of the head: inhale - elbows to push back. - Exhale - relax back.

8. Upright sitting: inhale - upper limbs out for the body - exhale - back, upper limbs change.

9. "Dumbbells": inhale, hands up - exhale, hands go by the elbows to the body, as if we had dumbbells

10. Brügger sitting position: inhale - upper limbs lift, exhale - to pull the knee to the chest.

11. "Blending" (left / right): the starting position is arms raised ahead, palms together, shoulders down. Inhale- hold arms close to the body, exhale - arms upward. Note: make a move as if you are mixing something in a big pot.

12. Brügger sitting position: inhale - put right / left hand, to the left/ right and down, body and head turn towards the hands, exhale - raise your hand and look at it.

Exercises to calm down

- Brügger sitting position: inhale - lift the upper limbs, exhale - back.

- Bending forward head: inhale - lift your hands, exhale - smooth, mild thoracic bend.

From the alternative methods we have used mudras. Mudras - involve the entire body, most are performed with the hands and fingers. We have used mudras for abdominal and thoracic breathing. 


\section{Results}

For the data analysis, we used the program Number Cruncher Statistical System (NCSS). After each examination, we indicated graphical representations (box plot) of the decomposition of results in pre-test and posttest, which is easy to see whether there was any expected change - improving or not. Pretest X-ray examination showed that the difference of vertical movement of the lower ribs during inhalation and exhalation was 2-35 mm (Figure 1). The vital capacity of the lungs was somewhat lower if unaffected. Measuring the circumference of the chest showed little difference between the indices (maximum inhalation and exhalation) indicating the excursion of the chest. These indices are equal to about $3 \mathrm{~cm}$, which means reduced chest excursion. Respiratory rate for all respondents with SCI was 13 to 22 breaths / $\min$.

After six months, we repeated the tests. X-ray examination showed that the difference in the movement of the lower ribs on the inhale and exhale increased by $49 \%$, or $6-45 \mathrm{~mm}$ (fig.1). Circumference of the chest during inspiration increased by $3.5 \%$ and chest circumference during exhalation decreased by $1.27 \%$. One second vital capacity increased by $5.68 \%$ and forced vital capacity of the lungs of $7.61 \%$. Respiratory rate decreased on average by $16.22 \%$.

Figure 1. The Radiograph of the Lungs Pre-test Post-test

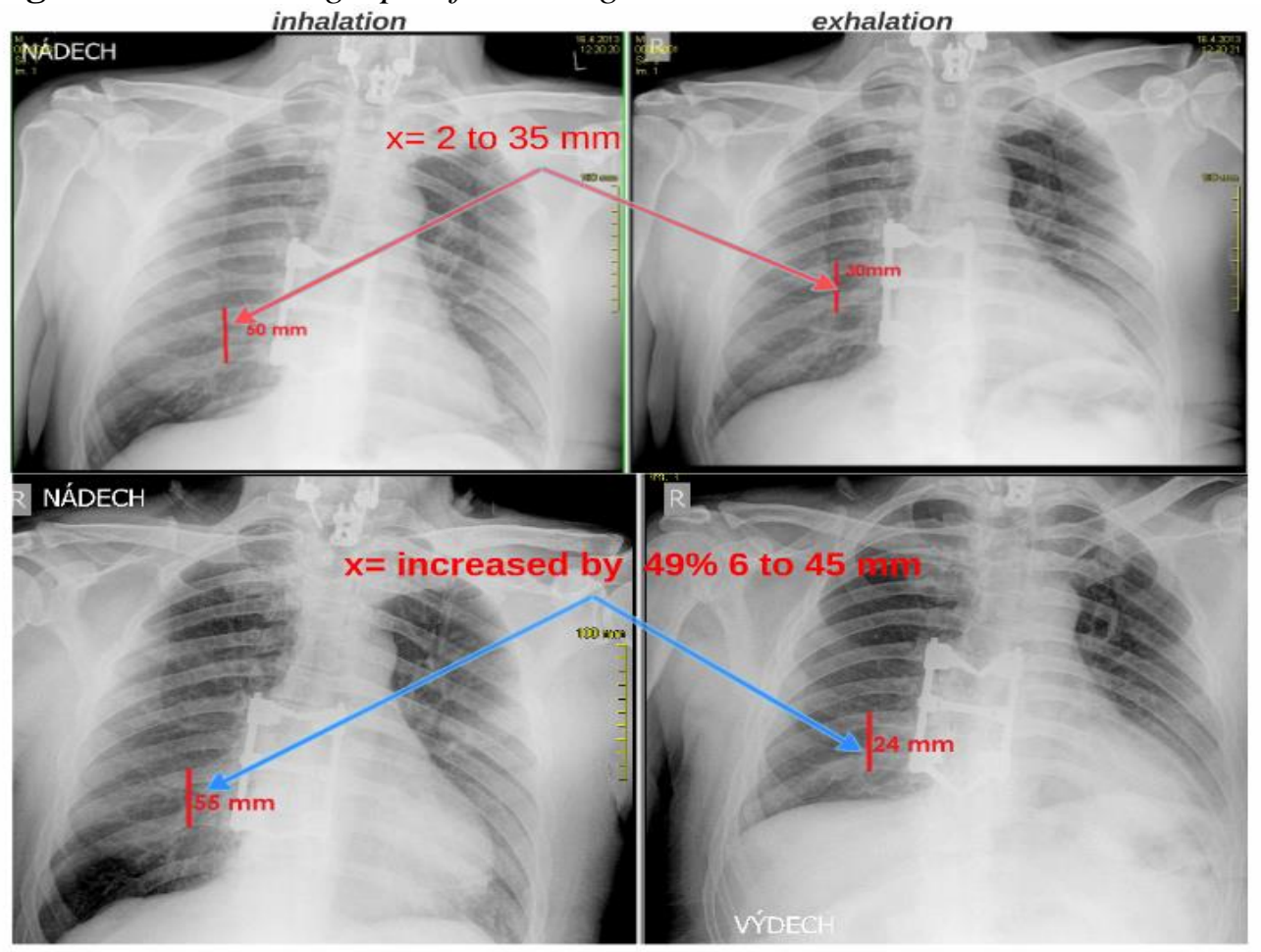




\section{Discussion}

This study showed that respiratory muscles in individuals with the SCI can be trained as in the healthy population. These findings are consistent with previous studies summarized by Brown (2006). The assumption that breathing exercises have a positive meaning for people with SCI was confirmed by our measurements. This is demonstrated by measuring the difference between the movement of the lower ribs during inhalation and exhalation, which was made by means of X-ray examination of the lungs. With these results, it can be assumed that the breathing exercises affect not only the intercostal muscles, but also other major respiratory muscles and accessory respiratory muscles. From the X-ray images it can be seen that there is increased movement of the diaphragm. From the above, we can conclude that it improves the strength of the diaphragm, as was in the study of Kogan (1996), and leads to a more active lifting of ribs, that have an influence on posture (Velé, 2006). It is a fact that respiration and posture have a clear relationship, which confirms our study and studies that we mentioned earlier (Chen,1990; Baydur, 2001; Gandevia, 2002; Makhsous, 2003).

This article was created with the support of the Ministry of Education VZ MSM 0021620864

\section{References}

Baydur, A., Adkins, RH., Milic-Emili, J. (2001). Lung mechanics in individuals with spinal cord injury: effects of injury level and posture. J Appl Physiol, 90: 405-11.

Brown, R., Anthony, F. (2006). Respiratory Dysfunction and Management in Spinal Cord Injury. , DiMarco MD, Jeannette D Hoit PhD CCC-SLP, and Eric Garshick MD MOH. Respir Care, 51(8), p. 853-868.

Chen, CF,. Lien, IN., Wu MC. (1990). Respiratory function in patients with spinal cord injuries: effects of posture. Paraplegia, 28: 81-6.

Dyson-Hudson, TA., Kirshblum, SC. ( 2004). Shoulder pain in chronic spinal cord injury. Part I: Epidemiology, etiology, and pathomechanics. J Spinal Cord Med, 27: 4-17.

Gandevia SC, Butler JE, Hodges PW, Taylor JL. (2002). Balancing acts: respiratory sensations, motor control and human posture. Clin Exp Phar-macol Physiol, 29: $118-121$.

Gironda, RJ., Clark, ME., Neugaardm, B., Nelson, A. (2004). Upper limb pain in a national sample of veterans with paraplegia. J Spinal Cord Med, 27: 120-7.

Hobson, DA., Tooms, RE. (1992). Seated lumbar/pelvic alignment. A comparison between spinal cord-injured and noninjured groups. Spine, 17: 293-8.

Jackson, AB., Grooms, TE. (1994).Incidence of respiratory complications following spinal cord injury. Arch Phys Med Rehabil, 75: 270-5.

Kogan, I., McCool, FD., LibermanI, SL., Garshick, E., Shannon, K., Frisbee, JH., Brown, R. (1996). Diaphragm hypertrophy during inspiratory muscle training in tetraplegia (abstract). Am J Respir Crit Care Med, 153(4): A25.

Lalloo, UG., Becklake, MR., Goldsmith, CM. (1991). Effect of standing versus sitting position on spirometric indices in healthy subjects. Respiration, 58: 122-5. 
Makhsous, M., Lin, AF., Hendrix, RW., Hepler, M., Zhang, LQ. (2003). Sitting with adjustable ischial and back supports: biomechanical changes. Spine, 28:1113-21

McKinley, WO., Jackson, AB., Cardenas, DD., DeVivo, MJ. (1999). Longterm medical complications after traumatic spinal cord injury: a regional model systems analysis. Arch Phys Med Rehabil, 80: 1402-10.

Rodgers, MM., McQuade, KJ., Rasch, EK., Keyser, RE., Finley, MA. (2003). Upperlimb fatigue-related joint power shifts in experienced wheelchair users and non wheelchair users. JRehabil Res Dev, 40:27-37.

Thorfinn, J., Sjöberg, F., Lidman, D. (2002). Sitting pressure and perfusion of buttock skin in paraplegic and tetraplegic patients, and in healthy subjects: a comparative study. Scand J Plast Reconstr Surg Hand Surg, 36: 279-83.

Van Houtte, S., Vanlandewijck, Y,. Gosselink, R. (2006). Respiratory muscles training in person with spinal cord injury: A systematic review. Respiratory Medicine, 100, 1886-1895.s

Velee, F. (2006), Kinesiology, Overview of Clinical Kinesiology and Pathokinesiology for Diagnosis and Therapy of Movement Disorders System, Prague, Triton. 
